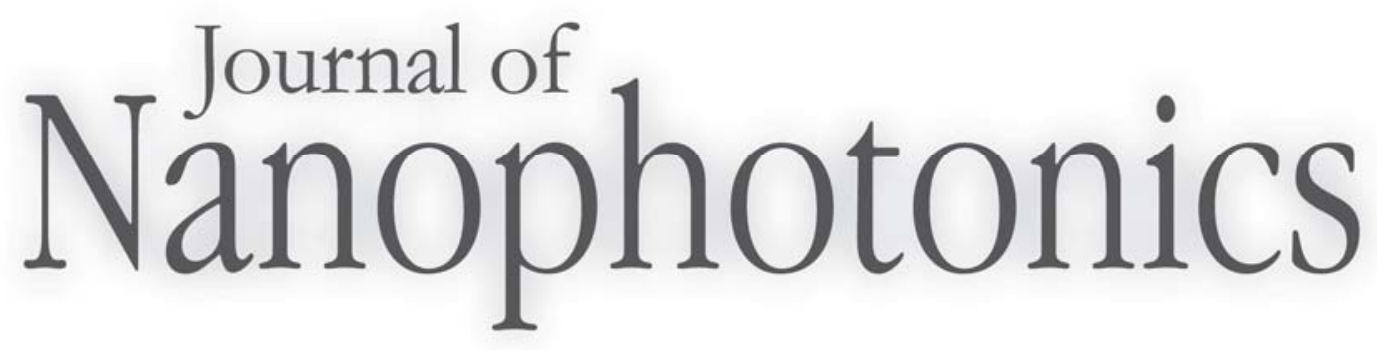

SPIEDigitalLibrary.org/jnp

\title{
Modeling the scaling law of surface plasmon resonance in gold spherical nanoshells
}

Jui-Teng Lin 


\title{
Modeling the scaling law of surface plasmon resonance in gold spherical nanoshells
}

\author{
Jui-Teng Lin \\ National Taiwan University, Institute of Photonic and Optoelectronics, Taipei, Taiwan 10617 \\ Currently with: New Vision, Inc. Taipei, Tawian \\ jtlin55@gmail.com
}

\begin{abstract}
Analytic formulas for spherical nanoshell (Au/silica) resonance wavelength and refractive index sensitivity were derived and compared with a numerical nonlinear theory. A universal scaling law was deduced in terms of a normalized thickness defined by the ratio of the shell thickness and its core diameter. The calculated figure of merit shows a maximum at an optimal value of the resonance wavelength and normalized thickness. The nonlinear theory of nanoshells improves the accuracy of the linear theory in the short wavelength regime (500 to $650 \mathrm{~nm})$.
\end{abstract}

Keywords: nanoshell; nanosensor; sensitivity; gold; silica; scaling law.

\section{INTRODUCTION}

Bioimaging, biosensing, drug delivery, diagnostics, and selective phototherapeutics have been the focus of applications of metal nanoparticles in biomedicine [1-4]. Various nanoparticles have been explored for the use of surface plasmon resonance (SPR), including shapes in spheres, rods, boxes, cages, and shells [4-8]. By changing the shape of nanoparticles from spheres to nanorods, the absorption and scattering peaks change from visible to the nearinfrared (NIR) regime. Compared to visible light, light in the NIR regime offers the advantages of larger absorption and scattering cross sections, and much deeper penetration depth in tissues [1-5]. The red-shift of the absorption peak in nanorods is governed by the aspect ratio (defined as the ratio of the length to the cross sectional diameter), whereas it is governed by the shell thickness in nanoshells [6].

The performance of nanosensors is characterized by not only the index sensitivity $(M)$, but more importantly, the figure of merit FOM $=M / F W H M$, where FWHM is the full width at half maximum of the extinction profiles of the nanoparticles. The index sensitivity defined by the red-shift of the absorption peak per unit change of the refractive index of the sensing medium has been studied for nanoparticles of various shapes $[9,10]$.

This work presents the nonlinear theory for the resonance wavelength, which is numerically solved as a function of the core and shell diameter ratio $(r / R)$, and the refractive index of the core and sensing medium. Compared to the nonlinear theory, the previous linear theory $[3,4,6,9,10]$ suffers large errors, particularly for short wavelengths $(<650 \mathrm{~nm})$ and/or large ratios, $r / R>0.3$. A scaling law based on a universal parameter defined by a normalized thickness of the shell is introduced. Finally, the sensitivity and the FOM are calculated, showing an optimum normalized thickness.

\section{THEORY}

As shown in Fig. 1, a spherical nanoshell is defined by its core diameter (r), outer diameter (R) coated by a thin gold layer with a thickness $t=R-r$, the refractive index of the core (n1), and the medium (n). Application of the quasistatic approximation (for nanoshells with diameters less than about $50 \mathrm{~nm}$ ) to the Lorenz-Mie scattering theory indicates that the resonance wavelength $(\lambda)$ is the solution of $[5,6]$ 


$$
\begin{gathered}
T=(1+a / b)^{-1 / 3}-1, \\
a=1.5 \varepsilon^{\prime}(\lambda)\left(n_{1}^{2}+2 n^{2}\right), \\
b=\left[\varepsilon^{\prime}(\lambda)\right]^{2}-\left(n_{1}^{2}+n^{2}\right) \varepsilon^{\prime}(\lambda)+n_{1}^{2} n^{2}-\left[\varepsilon^{\prime \prime}(\lambda)\right]^{2},
\end{gathered}
$$

where a universal parameter, given by the normalized thickness $T=t / r$, is introduced. The prior scaling equation, however, shows that $T$ is a nonlinear function of the resonance wavelength $(\lambda)$. An explicit scaling equation of the resonance wavelength as a function of $T$ will be found. In general, Eq. (1) is good for metallic shells. This study focuses on the gold nanoshell.

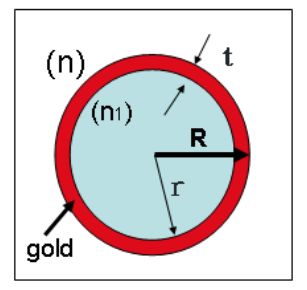

Fig. 1 The geometry of a nanoshell defined by its core inner $(r)$ and outer diameter $(R)$ coated by a thin layer of gold with thickness $t=R-r$. Also shown are the refractive index of the core $\left(n_{1}\right)$ and the medium $(n)$.

The real $\left(\varepsilon^{\prime}\right)$ and imaginary part $\left(\varepsilon^{\prime \prime}\right)$ of gold relative permittivity, reported by Johnson and Christy [7], can be fit to the following nonlinear equations:

$\varepsilon(\lambda)=12.5-0.02 \lambda-0.0000333 \lambda^{2}$, for wavelength range of (550 to 2000$) \mathrm{nm}$,

$\mathcal{E}^{\prime \prime}(\lambda)=0.00005 \lambda^{2}-0.071 \lambda+25.65$, for short wavelength range of (500 to 700$) \mathrm{nm}$,

$\varepsilon^{\prime \prime}(\lambda)=0.000011 \lambda^{2}-0.0127 \lambda+4.6$, for long wavelength $>700 \mathrm{~nm}$.

As described by Eq. (1), the resonance wavelength $(\lambda)$ is highly nonlinear and can only be solved numerically. An analytic formula is available only if one ignores the $\left(\varepsilon^{\prime \prime}\right)^{2}$ term in Eq. (3). This linear approximation was used in earlier work $[3,6]$ that suffers errors, particularly for short wavelengths $(<650 \mathrm{~nm})$ and/or large $T>0.3$, in which the contribution from $\left(\varepsilon^{\prime \prime}\right)^{2}$ cannot be ignored.

Given the scaling equation for the resonance wavelength as a function of the sensing medium refractive index $(n)$ and $T$, one can calculate the sensitivity function by its red-shift rate per unit change of $n$, defined by $M=\mathrm{d} \lambda / \mathrm{d} n$.

\section{RESULTS AND DISCUSSIONS}

\subsection{Resonance wavelength}

The resonance wavelength $(\lambda)$ is calculated by the numerical solution of Eq. (1) and is shown in Fig. 2 as a function of the diameter ratio $(r / R)$ for various sensing medium refractive indices of $n=1.33$ to 1.50 . These curves show that the resonance wavelength red-shift increases for larger $r / R$ ratios, or thinner shells. Furthermore, for a given $r / R$ ratio, the resonance wavelength is also red-shifted for a larger sensing medium refractive index $(n)$. Figure 2 is plotted from Eq. (1), noting that $r / R=1 /(1+T)$. 


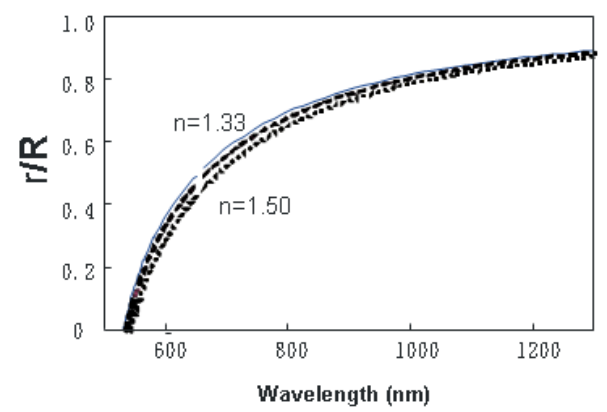

Fig. 2 The resonance wavelength $(\lambda)$ versus the diameter ratio $(r / R)$ for various sensing medium refractive indices $n=1.33,1.40$, and 1.50 , for a gold nanoshell with a silica core (with $n_{1}=1.45$ ).

\subsection{Error of linear approximation}

As mentioned earlier, the imaginary part $\left(\varepsilon^{\prime \prime}\right)$ of Eq. (3) was ignored in the linear approximation used by earlier work [3], and errors occur particularly for short wavelengths $(<650 \mathrm{~nm})$ and/or large $T>0.3$. Examples are shown in Figs. 3 and 4 comparing our exact nonlinear calculations (solid curves) and the linear approximation (dashed curves), which is valid only in small $T<0.3$.

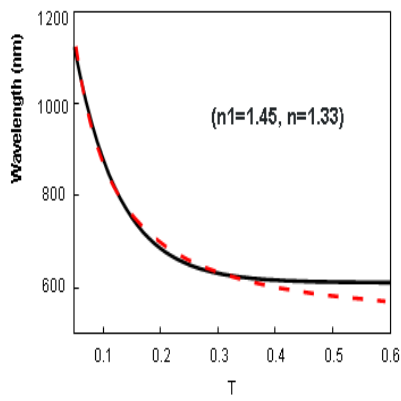

Fig. 3 The resonance wavelength versus the normalized thickness $T$ for nanoshell $\mathrm{Au} / \mathrm{silica}$ in water medium $(n=1.33)$, where the solid curve is the exact and the dashed is the approximate.

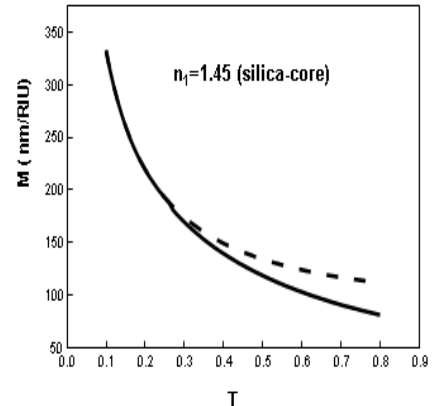

Fig. 4 The sensitivity function $(M)$ defined by the slope of the curves in Fig. 3.

\subsection{Scaling law}

As defined earlier, a universal parameter is given by the normalized thickness $T=t / r$. This definition is based on the fact that, as shown by Eq. (1), the resonance wavelength is solely related to the ratio of $T=t / r$ rather than the individual values of $r, R$, or the thickness $(t)$. Universal scaling was also presented by Jain and El-Sayed [3] in 2007 based on their numerical fit. However, their claimed new discovery can be readily seen just by Eq. (1), which was explored much early in 1999 [6]. In addition, their fit equation based on a linear approximation will suffer errors for the regime of $T>0.3$, as shown by Figs. 3 and 4 . All the calculations in this study are based on the exact nonlinear equation of Eq. (1).

It is worthy to note that the concept of a scaling law governed by a universal parameter in general could be applied to various nanoparticle structures. For nanoshells, it is defined as the normalized thickness $T=t / r$; for nanorods, it is given by the aspect ratio (length/width); and in nanofibers, it is defined as the ratio of fiber length and its core diameter. 
Figure 5 shows the strong dependence of the resonance wavelength on both the shell thickness $(t)$ and its size $(R)$, if one plots the curves against $t$ or $R$. However, as shown in Fig. 6 , the resonance wavelength curves are universally scaled by the normalized parameter $T=t / r$. In contrast to Fig. 5, the resonance wavelength curves in Fig. 6 are not influenced by the individual values of $r$ and $R$, but only depend on their ratio $r / R$. Figure 6 can be also compared with Fig. 2, noting that $T=R / r-1$.

For silica-core $\left(n_{1}=1.45\right)$ gold shells in water medium $(n=1.33)$, the curve in Fig. 6 can be fit to an exponential decay scaling law as follows:

$$
\lambda=a+b \exp (-T / c),
$$

with $a=568.2, b=761.5$, and $c=0.11117$, which is more accurate than that of Jain and El-Sayed [3] ignoring the nonlinear term in Eq. (3). It should be noted that the scaling fit coefficients $(a, b, c)$ are slightly influenced by the refractive index of the core and the sensing medium, as shown by Fig. 6 .

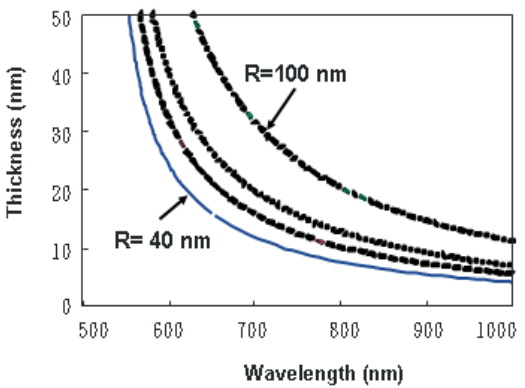

Fig. 5 Shell thickness $(t)$ versus resonance wavelength for various shell sizes $R=(40,60,80,100) \mathrm{nm}$ in Au/silica with sensing medium refractive index $n=1.33$.

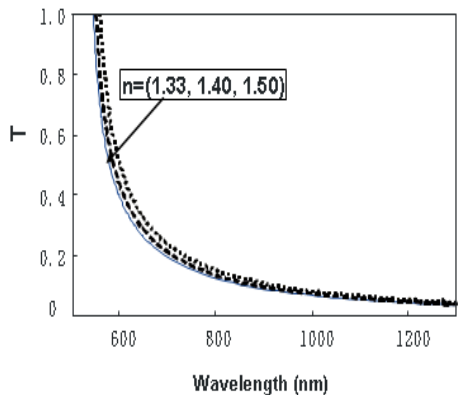

Fig. 6 Shell normalized thickness $(T)$ versus resonance wavelength for various medium refractive index $n=(1.33,1.4$, 1.5).

\subsection{Sensitivity}

To calculate the sensitivity function $(M)$ defined by $M=\mathrm{d} \lambda / \mathrm{d} n$, one needs to calculate the resonance wavelength versus the sensing medium ( $n$ ), which is calculated from Eq (1) and shown in Fig. 7 for various normalized thicknesses (T). The curves for $M$ versus the resonance wavelength, shown by Fig. 8, are calculated from the slopes of Fig. 7. It should be noted that higher sensitivity is available for smaller $T$, or thinner shells for a given shell size $(R)$. However, a thin shell alone does not guarantee higher sensitivity. For example, $(t, r)=$ $(0.05,25),(0.1,50)$, and $(0.2,100)$ all have the same $M$ value according to our scaling law, since they have the same ratio $T=(t / r)=0.02$.

For the special case of hollow shells, with $n_{1}=n=1.0$, or with index-matched interior and exterior $n_{1}=n$, the analytic formula for $M$ is available under the linear approximation of Eq. (1) $\varepsilon^{\prime}=-2 X n^{2}$, where $X$ is a shape-dependent factor given by $X=(1-L) / L$, and the shape parameter $L$ equals $1 / 3$ for a sphere [5]. By further using the linear relation for gold relative permittivity [8], $\mathcal{E}^{\prime}=-0.071 \lambda+33.05$, the $M$ function for hollow shells is analytically given by $M=(2 / n)(\lambda-465.5)$, which shows that $M$ is linearly proportional to the resonance wavelength $(\lambda)$ and inversely proportional to the medium refractive index, but independent to the shell structure (thickness or size) and its core refractive index. 


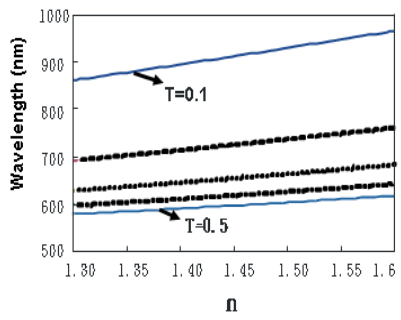

Fig. 7 Resonance wavelength versus sensing medium refractive index $(n)$ for various normalized thicknesses $(0.1,0.2$, $0.3,0.4,0.5)$.

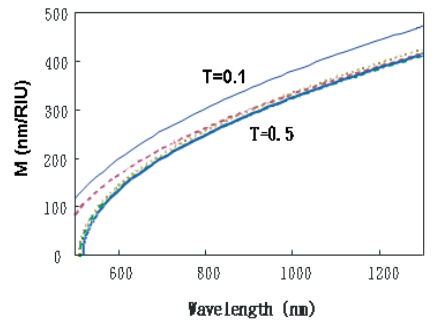

Fig. 8 The sensitivity function $(M)$ versus resonance wavelength calculated from the slopes of the curves in Fig. 8.

However, for spherical nanoshells, in general, the $T$-dependence of $M$ can be fit to an exponential decaying function

$$
M=a^{\prime}+b^{\prime} \exp \left(-T / c^{\prime}\right),
$$

where $a^{\prime}=127.68, b^{\prime}=596.18$, and $c^{\prime}=0.1023$ for a silica core $\left(n_{1}=1.45\right)$ in water medium ( $n=1.33$ ). One can also derive Eq. (5) from the derivative of Eq (4) with respect to the medium refractive index $(n)$ evaluated at $n=1.33$.

For resonance wavelength range in the near-infrared of (700 to 1000) $\mathrm{nm}, M=(250$ to 400) (nm/RUI) in Au/silica nanoshells, as shown by Fig. 8. This calculated sensitivity of nanoshells is comparable to that of gold nanorods (300 to 600) (nm/RIU) [8].

\subsection{Figure of merit}

The figure of merit (FOM) for a nanoshell is defined by its sensitivity divided by its spectral broadening, the FWMH, or FOM $=M / F W H M$. Using the FWHM of gold, the FOM versus $T$ is shown in Fig. 9, which indicates a maximum at an optimal diameter ratio $(r / R)$ around 0.85 , corresponding to the resonance wavelength around $800 \mathrm{~nm}$ and the normalized thickness $(T)$ around 0.1. It is worthy to note that the optimal feature of FOM in nanoshells is also found in gold nanorods [8] with an optimal aspect ratio (defined by its length and width) of (3.5 to 4.0). Combining the calculated data shown by the curves of Figs. 6 and 10, a high FOM of (6.0 to 8.0) is achievable in biosensors using a NIR laser of (700 to $900 \mathrm{~nm}$ ). In comparison, lasers in the visible spectrum of 550 to $700 \mathrm{~nm}$, have lower FOM of 3.0 to 4.0. The NIR lasers are also better candidates for biosensnors and selective phototherapeutics when deep tissue penetration is required.

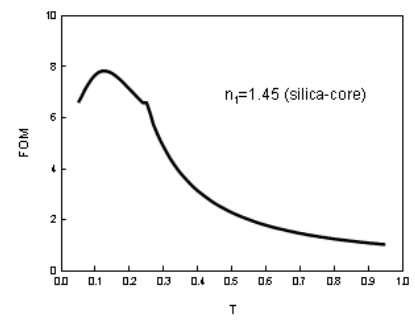

Fig. 9 Figure of merit (FOM) versus the nanoshell normalized thickness $(T=t / r)$ for a silica core $\left(n_{1}=1.45\right)$ and in water medium $(n=1.33)$.

\section{CONCLUSION}

Scaling laws in Eqs. (4) and (5) for resonance wavelength and refractive index sensitivity, respectively, are derived based on a nonlinear theory and are fit to the numerical results. A normalized thickness $(T)$ defined by the ratio of the shell thickness and its core diameter is 
introduced. The calculated figure of merit shows a maximum at an optimal value of the resonance wavelength (around $800 \mathrm{~nm}$ ), diameter ratio $(r / R)$ around 0.85 , and $T$ around 0.1 . It is predicted by this study that the high figure of merit (FOM) and deep tissue penetration of NIR lasers offer many advantages over visible lasers.

\section{Acknowledgments}

This work is partially supported by the National Science Council (NSC) of Taiwan, (grant number NSC 98-3011-P-002-002). The author thanks the assistance from Yu-Ling Hong for figure drawing. The author also thanks many suggestions from the reviewer.

\section{References}

[1] L.Tong, G. Wei, and J.X. Cheng, "Gold nanorods as contrast agents for biological Imaging: optical properties, surface conjugation and photothermal effects," Photochem. Photobiol. 85, 21-32 (2009) [doi:10.1111/j.1751-1097.2008.00507].

[2] J.L. West and N. J. Halas, "Engineered nanomaterials for biophotonics applications:improving sensing,imaging, and therapeutics," Annu. Rev. Biomed. Eng. 5, 285-292 (2003) [doi:10.1146/annurev.bioeng.5.011303.120723].

[3] P. K. Jain and M. A. El-Sayed, "Surface plasmon resonance sensitivity of metal nanostructures: physical basis and universal scaling in metal nanoshells," J. Phys. Chem. C 111, 17451-17454 (2007) [doi: 10.1021/jp0773177].

[4] H. Chen, X. Kou, Z. Yang, W. Ni, and J. Wang, "Shape- and size-dependent refractive index sensitivity of gold nanoparticles, “ Langmuir 24, 5233-5237 (2008) [doi:10.1021/la800305j].

[5] C.F. Bohren and D. R. Huffman, Absorption and Scattering of Light by Small Particles, Wiley, New York (1983).

[6] R. D. Averitt, S. L. Westcott, and N. J. Halas, "Linear optical properties of gold nanoshells,” J. Opt. Soc. Am. B16, 1824-832(1999) [doi:10.1364/JOSAB.16.001824].

[7] P. B. Johnson and R.W. Christy, "Optical constants of the noble metals," Phys. Rev. B 6, 4370-4379 (1972) [doi:10.1103/PhysRevB.6.4370].

[8] J. T. Lin and Y. L. Hong, "A nonlinear optical theory for gold nanorods," Proc. SPIE 7574, 75740A1-5 (2010) [doi:10.1117/12.154577].

[9] .M. M. Miller and A. A. Lazarides, "Sensitivity of metal nanoparticles surface plasmon resonance to the dielectric environment," J. Phys. Chem. B 109, 2155621565 (2005) [doi:10.1021/jp054227y].

[10] M. Cao and N. Gu, "Optimized surface plasmon resonance sensitivity of gold nanoboxes for sensing applications," J. Phys. Chem. C 113, 1217-1221 (2009) [doi: 10.1021/jp808000x].

Jui-Teng Lin received his $\mathrm{PhD}$ in chemical physics from the University of Rochester in 1980. He is currently the Chairman of New Vision, Incorporated (Taipei, Taiwan). He was a visiting professor at National Chiao-tung University and National Taiwan University (2007 to 2010), a faculty member at CREOL,University of Central Florida (1986 to 1991), and founder and CEO of NASDAQ companies (1991 to 2004). He holds more than 30 international patents and is the inventor of the LASIK procedure using flying-spot scanning lasersthat is currently used in all major eye hospitals worldwide. He has published more than 120 book chapters and journal papers. His achievements include Who's Who in Leading American Executives (1993), Model of Overseas Chinese Young Entrepreneur (1997), and chair and/or cochair at SPIE conferences. He is a Fellow of the American Society of Laser Medicine and Surgery (ASLMS). 